\title{
SELF 2-DISTANCE GRAPHS
}

\author{
A. AZIMI AND M. FARROKHI D. G.
}

\begin{abstract}
All finite simple self 2-distance graphs with no 4-cycle, diamond, or triangles with a common vertex are determined. Utilizing these results, it is shown that there is no cubic self 2-distance graphs.
\end{abstract}

\section{INTRODUCTION}

Let $(X, \rho)$ be a metric space and $D$ be a set of positive real numbers. The distance graph $G(X, D)$ of $X$ with respect to a distance set $D$ is the graph whose vertex set is $X$ and two distinct vertices $x$ and $y$ are adjacent if $\rho(x, y) \in D$.

The well-known unit distance graph $G\left(\mathbb{R}^{2},\{1\}\right)$ is the first instance of a distance graph arising from a question of Edward Nelson about its chromatic number in 1950 (see [11, Chapter 3]). It is shown by Nelson and Isbell [5], Moser and Moser 8] and Hadwiger, Debrunner and Klee [4] that the chromatic number of this graph is between 4 and 7. Unit distance graphs are also investigated on any of the sets $\mathbb{R}^{n}, \mathbb{Q}^{n}$ and $\mathbb{Z}^{n}$ as well (see [1] for a detailed history). The other well-studied sort of distance graphs are the distance graphs $G(\mathbb{Z}, D)$ introduced by Eggleton, Erdös and Skiltons in [3, where $D$ is a set of positive integers. Clearly, every graph $\Gamma$ with associated distance function $d$ defines a metric space $(\Gamma, d)$. Hence, we may define the distance graphs of the graph $\Gamma$ with respect to a set of positive integer distances. For example, the $n$th power of a graph $\Gamma$ is defined simply as the distance graph $G(\Gamma,\{1, \ldots, n\})$. We refer the interested reader to the survey articles [2, 7, 6, for further details concerning the mentioned three kinds of distance graphs, respectively.

The $n$th distance graph (or $n$-distance graph) of a graph $\Gamma$ is defined simply as $\Gamma_{n}:=G(V(\Gamma),\{n\})$. The study of $n$th distance graph initiated by Simić [10] while solving the graph equation $\Gamma_{n} \cong L(\Gamma)$, where $L(\Gamma)$ is line graph of $\Gamma$. Regarding the same problem, we have classified of all graphs whose 2-distance graphs are path or cycle in [1].

A graph is said to be self $n$-distance graph if it is isomorphic to its $n$-distance graph. The aim of this paper is to investigate self 2-distance graphs under some conditions. More precisely, we will show that self 2-distance graphs with no squares or disjoin triangles are either odd cycles of order $\geq 5$ or the edge product $C_{5} \mid C_{3}$. Also, we show that a self 2-distance graph with no diamond is either an odd cycle of order $\geq 5$, the edged product $C_{5} \mid C_{3}$, or it is isomorphic to one of graphs in Figures 5.1 .1 or 5.1.2. One note that our knowledge about $n$-distance graphs can be used to answer/pose some problems in groups through their Cayley graphs. Indeed, we may observe that the $n$th distance graph of a Cayley graph Cay $(G, S)$ of $G$ equals Cay $\left(G, S^{n} \backslash S\right)$ and hence it is itself a Cayley graph. Any isomorphism between

2000 Mathematics Subject Classification. Primary 05C12; Secondary 05C60, 05C76.

Key words and phrases. Distance graph, regular graph, forbidden subgraph. 
$\operatorname{Cay}(G, S)$ and $\operatorname{Cay}\left(G, S^{n} \backslash S\right)$ give the constraint $\left|S^{n}\right|<2|S|$ on $S$, the problem which is the subject of resent research. On the other hand, such an isomorphism brings us the question whether $S^{n} \backslash S$ and $S$ are conjugate via an automorphism of $G$, which is a central problem in the theory of Cayley graphs. In case $S^{n} \backslash S=S^{\theta}$ for some $\theta \in \operatorname{Aut}(G)$, we have obviously $\operatorname{Cay}\left(G, S^{n} \backslash S\right) \cong \operatorname{Cay}(G, S)$, that is, $\operatorname{Cay}(G, S)$ is a self $n$th distance graph.

Throughout this paper, we use the following notations: The maximum degree of vertices of a graph $\Gamma$ is denote by $\Delta(\Gamma)$ and $N_{\Gamma}(v)$ illustrates the set of all neighborhoods of the vertex $v$ in $\Gamma$. Also, $\nabla(\Gamma)$ denotes the number of triangles in a graph $\Gamma$. All graphs in this papers are finite simple graphs with no multiple edges. Remind that a diamond is the edge product $\mathcal{D}=C_{3} \mid C_{3}$, where the edged product of two edge-transitive graphs $\Gamma_{1}$ and $\Gamma_{2}$ is obtained by identification of an edge from $\Gamma_{1}$ and $\Gamma_{2}$.

\section{Preliminary Results}

We begin with a simple query about the existence of self 2-distance graphs. Clearly, any odd cycle of length $\geq 5$ is a self 2 -distance graph. As we shall see later, odd cycles are exceptional examples in the class of all self 2-distance graphs. We note that the class of self 2-distance graphs is broad as Propositions 2.2 and 2.3 provide ample of them. The following simple key lemma plays an important role in our study.

Lemma 2.1. Let $\Gamma$ be a graph. Then $\operatorname{diam}(\Gamma)=2$ if and only if $\Gamma_{2}=\Gamma^{c}$.

Proposition 2.2. Let $\Gamma$ be a self-complementary graph with diameter two. Then $\Gamma_{2} \cong \Gamma$.

Proposition 2.3. Every graph is an induced subgraph of a self 2-distance graph.

Proof. Let $\Gamma$ be an arbitrary graph. Consider two disjoint copies $\Gamma_{1}$ and $\Gamma_{2}$ of $\Gamma$ and two disjoint copies $\Gamma_{3}$ and $\Gamma_{4}$ of $\Gamma^{c}$, and let $v$ be a new vertex. Then the graph with vertex set

$$
V\left(\Gamma_{1}\right) \cup V\left(\Gamma_{2}\right) \cup V\left(\Gamma_{3}\right) \cup V\left(\Gamma_{4}\right) \cup\{v\}
$$

and edge set

$$
E\left(\Gamma_{1}\right) \cup E\left(\Gamma_{2}\right) \cup E\left(\Gamma_{3}\right) \cup E\left(\Gamma_{4}\right) \cup E,
$$

where

$$
E=\left\{\left\{v, v_{1}\right\},\left\{v, v_{2}\right\},\left\{v_{1}, v_{3}\right\},\left\{v_{2}, v_{4}\right\},\left\{v_{3}, v_{4}\right\}: v_{i} \in V\left(\Gamma_{i}\right), i=1,2,3,4\right\}
$$

is a self 2-distance graph containing $\Gamma$ as a subgraph (see Figure 2.3.1).

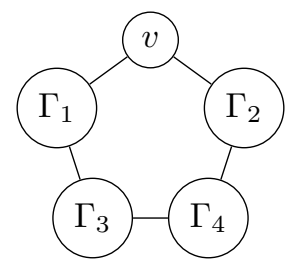

Figure 2.3.1

Lemma 2.4. If $\Gamma$ is a self 2-distance graph which is not an odd cycle, then $\operatorname{gr}(\Gamma)=$ 3 . 
Proof. Since $\Delta(\Gamma)>2$, we may choose a vertex $v$ of degree $\geq 3$. If $N_{\Gamma}(v)$ is not empty, then $\Gamma$ has a triangle. Thus we may assume that $N_{\Gamma}(v)$ is empty. But then $N_{\Gamma}(v)^{c}$ is a subgraph of $\Gamma_{2} \cong \Gamma$, which implies that $\Gamma$ has a triangle. Therefore $\operatorname{gr}(\Gamma)=3$.

The following lemma will be used in the next section.

Lemma 2.5. Let $\Gamma$ be a graph. Then

$$
|E(L(\Gamma))|=\left|E\left(\Gamma_{2}\right)\right|+|E(\Gamma)|+3 \nabla(\Gamma)-\left(\begin{array}{c}
|V(\Gamma)| \\
2
\end{array}\right)+\sum_{u \nsim v}\left|N_{\Gamma}(u) \cap N_{\Gamma}(v)\right| .
$$

In particular,

$$
|E(L(\Gamma))|=\left|E\left(\Gamma_{2}\right)\right|+3 \nabla(\Gamma)
$$

whenever $\Gamma$ has no 4-cycle subgraph.

Proof. It is straightforward.

\section{Graphs With No 4-CYCLE SUBGRAPH}

Throughout this section, we assume that $\Gamma \cong \Gamma_{2}$ is a graph with no 4-cycle as subgraph. Further, we assume that $\Gamma$ is not an odd cycle. A simple observation shows that every triangle in $\Gamma_{2}$ comes from an induced claw, an induces 6-cycle or an induced edge product $C_{5} \mid C_{3}$. Moreover, every 6 -cycle in $\Gamma$ is induced or it induces a graph isomorphic to $C_{5} \mid C_{3}$. To achieve the classification of graphs $\Gamma$ with the mentioned properties, we need to analyze the existence of some special subgraphs of $\Gamma$ as presented in Lemma 3.2 3.6. The following lemma will be used in the sequel without reference.

Lemma 3.1. $\Delta(\Gamma)=3$.

Proof. Since neither $\Gamma$ nor $\Gamma_{2}$ have 4-cycles and $N_{\Gamma}(v)^{c}$ is a subgraph of $\Gamma_{2}$ for all $v \in V(\Gamma)$, it follows that $\Delta(\Gamma) \leq 3$. Now, the fact that $\Gamma$ is not a cycle, implies that $\Delta(\Gamma) \geq 3$ so that $\Delta(\Gamma)=3$.

Lemma 3.2. If $\Gamma$ has a $C_{5} \mid C_{3}$ subgraph, then $\Gamma$ is isomorphic to $C_{5} \mid C_{3}$.

Proof. Suppose on the contrary that $\Gamma \not C_{5} \mid C_{3}$ and $S \subset V(\Gamma)$ induces a subgraph of $\Gamma$ isomorphic to $C_{5} \mid C_{3}$ (see Figure 3.1.1). Then there exists a vertex $v \in V(\Gamma)$ adjacent to some vertex of $S$. Clearly, $v$ is not adjacent to the temples for $\Delta(\Gamma)=3$.

First suppose that $v$ is adjacent to the forehead. If $v$ is adjacent to any of the jaws, then we get a 4 -cycle, which is a contradiction. Thus $N_{S}(v)=\{a\}$ or $\{a, d\}$, which imply that $\{v, b, d, f\}$ is a 4 -cycle in $\Gamma_{2}$, which is again a contradiction. Therefore $v$ is not adjacent to the forehead. Next assume that $v$ is adjacent to the chin. Clearly, $v$ is not adjacent to both $c$ and $d$, say $c$, for otherwise we have a $r$-cycle $\{c, d, e, v\}$. Bu then $\{a, f, e, v\} \subseteq N_{\Gamma_{2}}(c)$, that is, $\Delta\left(\Gamma_{2}\right)>3$, which is a contradiction. Finally, assume that $v$ is adjacent to any of the jaws. Then $v$ is adjacent to exactly one of the jaws, say $c$, for otherwise $\{v, c, d, e\}$ is a 4-cycle. Since $(S \cup\{v\})_{2} \not \models S \cup\{v\}$, there exists yet another vertex $u \in V(\Gamma) \backslash S \cup\{v\}$ adjacent to some vertex of $S \cup\{v\}$. If $u$ is adjacent to $v$, then either $N_{\Gamma_{2}}(c)$ contains $\{a, e, f, u\}$ as $u$ is cannot be adjacent to $c$, which is a contradictions. Thus $u$ is not adjacent to $v$ and by the same arguments as before $u$ is adjacent to one of the jaws. Since $u$ and $c$ are not adjacent, $u$ and $e$ must be adjacent, which implies that $\{b, f, u, v\} \subseteq N_{\Gamma_{2}}(d)$, a contradiction. The proof is complete. 


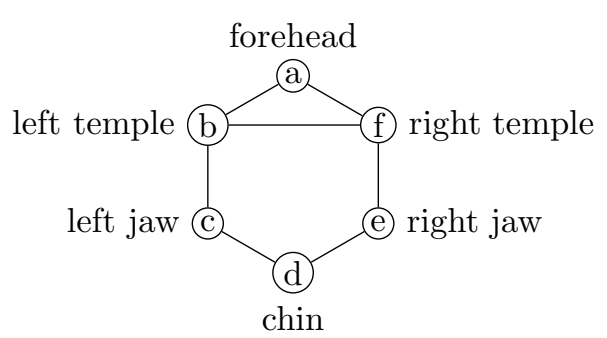

Figure 3.1.1

Lemma 3.3. If $\Gamma$ has a 5 -cycle, then $\Gamma$ is isomorphic to $C_{5} \mid C_{3}$.

Proof. Since $\Gamma ¥ C_{5}$, then there exist a vertex $v \in V(\Gamma) \backslash S$ adjacent to some vertex $u$ of $S$, where $S$ is a 5 -cycle in $\Gamma$. Clearly, $S$ is an induced subgraph of $\Gamma$. Let $a, b$ be two vertices adjacent to $u$ in $S$ and $c, d$ be two other vertices. Since $\Gamma$ has no 4-cycle it follows that $v$ is not adjacent to $c, d$. Now, it is easy to see that either $\Gamma$ or $\Gamma_{2}$ has a subgraph isomorphic to $C_{5} \mid C_{3}$, from which by Lemma 3.2. it follows that $\Gamma \cong C_{5} \mid C_{3}$.

Lemma 3.4. If $\Gamma$ has a 6-cycle, then $\Gamma$ is isomorphic to $C_{5} \mid C_{3}$.

Proof. If $\Gamma$ has a $C_{5} \mid C_{3}$ subgraph, then we are done. Thus we may assume that $\Gamma$ has no subgraph isomorphic to $C_{5} \mid C_{3}$. Let $S \subset V(\Gamma)$ denote a 6-cycle $a, b, c, d, e, f, a$ in $\Gamma$. Clearly, $S$ is an induced subgraph of $\Gamma$. Since $S_{2} ¥ S$, we have a vertex $u \in V(\Gamma) \backslash S$ adjacent to some vertex $a$ of $S$. Clearly, $u$ is adjacent to exactly one of $b, f$, say $b$, for otherwise either $\{b, d, f, u\}$ is a 4-cycle in $\Gamma_{2}$, or $\{b, a, f, u\}$ is a 4-cycle in $\Gamma$, which are both impossible. Again, the fact that $\Gamma$ has no 4-cycle implies that $u$ is not adjacent to $c, d, e, f$. Moreover, $u$ is the unique vertex adjacent to both $a, b$. Now, we have three cases:

Case 1. If $\Gamma$ has a subgraph $T$ as drawn in Figure 3.3.3, then $T$ is an induced subgraph and a simple verification shows that $T$ is a connected component of $\Gamma$, which implies that $\Gamma=T$. But then $\Gamma_{2} \nsucceq \Gamma$, which is a contradiction.

Case 2. If $\Gamma$ has a subgraph $T$ as drawn in Figure 3.3.2, then since $T_{2} \nsucceq T, \Gamma$ has a vertex $w^{\prime}$ adjacent to some vertex of $T$. If $w^{\prime}$ is adjacent to any of the vertices $a^{\prime}, b^{\prime}, c^{\prime}, d^{\prime}, u^{\prime}, v^{\prime}$, then we get a vertex of degree $\geq 4$ in $\Gamma$ or $\Gamma_{2}$, which is impossible. Thus $w^{\prime}$ is adjacent to $e^{\prime}$ or $f^{\prime}$ and by the previous argument it follows that $w^{\prime}$ is adjacent to both $e^{\prime}$ and $f^{\prime}$, which is impossible by Case 1 .

Case 3. $\Gamma$ has no subgraphs isomorphic to that of Figure 3.3.2. Then $u$ is the only vertex of $\Gamma$ adjacent to $S$ (see Figure 3.3.1). Since $(S \cup\{u\})_{2} \nsucceq S \cup\{u\}$, there exists a vertex $v \in V(\Gamma) \backslash S \cup\{u\}$ adjacent to $u$. But then $(S \cup\{u, v\})_{2}$ is an induced subgraph of $\Gamma_{2} \cong \Gamma$ isomorphic to the graph in Figure 3.3.4, from which it follows that $\operatorname{deg}_{\left(\Gamma_{2}\right)_{2}}(u) \geq 4$, a contradiction. 


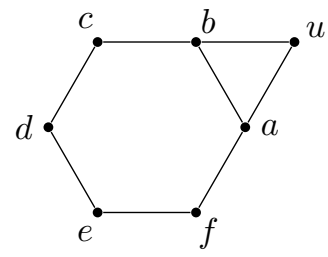

Figure 3.3.1

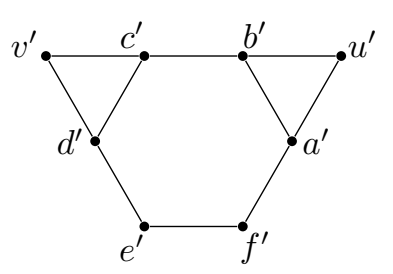

Figure 3.3.2

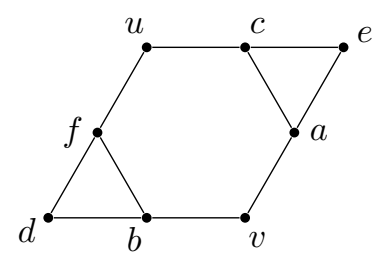

Figure 3.3.4

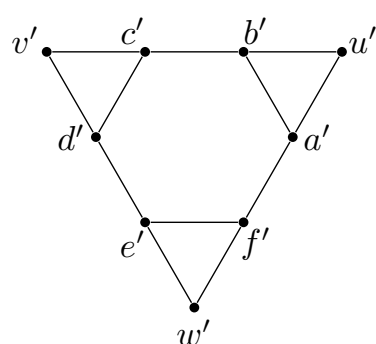

Figure 3.3.3

Lemma 3.5. If $\Gamma$ is not isomorphic to $C_{5} \mid C_{3}$, then $\Gamma$ has no cycles of length exceeding three.

Proof. By Lemmas 3.3 and 3.4 and hypothesis, $\Gamma$ has no cycles of lengths 4,5 or 6 . We proceed by induction to show that $\Gamma$ has no cycles of lengths $\geq 4$. Suppose $\Gamma$ has no cycles of lengths $4,5, \ldots, n$ for some $n \geq 6$. If $\Gamma$ has an $(n+1)$-cycle $C$, then $C$ is an induced subgraph of $\Gamma$. If $n+1$ is even, then clearly $\Gamma_{2}$ has two $(n+1) / 2$-cycles, which is a contradiction. Thus $n+1$ is odd. Since $\Gamma$ is not an odd cycle, there exists a vertex $v \in V(\Gamma)$ adjacent to some vertex $a \in V(C)$. Let $N_{C}(a)=\{b, c\}$. If $v$ is adjacent to some vertex in $C \backslash\{a, b, c\}$, then we obtain a cycle of length $l$ $(4 \leq l \leq n)$, which is a contradiction. If $v$ is not adjacent to $b, c$, then $\Gamma \cong \Gamma_{2}$ has a subgraph isomorphic to $(C \cup\{v\})_{2}$ that is an $|C|$-cycle with two adjacent vertices having a common neighbor. Hence, we may assume that $v$ is adjacent $b$ or $c$, say $b$. Since $\Gamma$ has no 4-cycle, $v$ is not adjacent to $c$. Let $N_{C}(b)=\{a, d\}$. Then $c, v, d$ is a path of length two in $\Gamma_{2}$. On the other hand, since $C_{2}$ is a subgraph of $\Gamma_{2}$, there is a path of length at most $n / 2$ from $c$ to $d$ disjoint from $c, v, d$. Hence $\Gamma_{2}$ has a cycle of length $l$ such that $4 \leq l \leq n / 2+2 \leq n$, which is a contradiction. The proof is complete.

Lemma 3.6. Triangles in $\Gamma$ have disjoint vertices.

Proof. If two triangles of $\Gamma$ have some vertices in common, then either $\Gamma$ or $\Gamma_{2}$ has a 4-cycle, which is a contradiction.

Now, we are ready to prove the main result of this section. To end this, we use the notion of distance between two subgraphs of a graph as the length of the shortest path connecting a vertex of the first subgraph to a vertex of the second subgraph.

Theorem 3.7. Let $\Gamma$ be a self 2-distance graph with no 4-cycle. Then either $\Gamma$ is an odd cycle or it is the edged product $C_{5} \mid C_{3}$. 


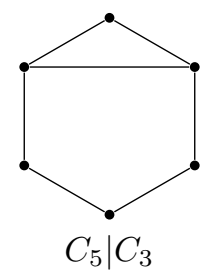

Proof. Let $\Gamma^{\prime}$ be the graph obtained from $\Gamma$ by contracting all triangles into single vertices. By Lemmas 3.5 and 3.6, $\Gamma^{\prime}$ is a tree. Let $v$ and $v^{\prime}\left(e\right.$ and $\left.e^{\prime}\right)$ be the number of vertices (edges) of $\Gamma$ and $\Gamma^{\prime}$, respectively. Also, let $n_{i}$ be the number of vertices of degree $i$ in $\Gamma$ for $i=1,2,3$. Clearly, $v^{\prime}=v-2 \nabla(\Gamma)$ and $e^{\prime}=e-3 \nabla(\Gamma)$. Since $\Gamma^{\prime}$ is a tree, we have $e^{\prime}=v^{\prime}-1$, which implies that $\nabla(\Gamma)=e-v+1$. On the other hand, by Lemma 2.5. $e_{L}-e=3 \nabla(\Gamma)$, where $e_{L}$ is the number of edges of $L(\Gamma)$, the line graph of $\Gamma$. Now, we have

$$
\begin{aligned}
|V(\Gamma)| & =n_{1}+n_{2}+n_{3}, \\
|E(\Gamma)| & =\frac{1}{2} \sum_{v \in V(\Gamma)} \operatorname{deg}_{\Gamma}(v)=\frac{n_{1}+2 n_{2}+3 n_{3}}{2}, \\
|E(L(\Gamma))| & =\sum_{v \in V}\left(\begin{array}{c}
\operatorname{deg}_{\Gamma}(v) \\
2
\end{array}\right)=n_{2}+3 n_{3},
\end{aligned}
$$

from which it follows that $n_{1}=3$.

If $\Gamma$ has no triangles then $\Gamma$ is a tree so that $\Gamma_{2}$ is disconnected, which is a contradiction. Hence $\Gamma$ has some triangles. A triangle in $\Gamma$ is said to be $i$-tailed if it contains $i$ cubic vertices. Clearly, $\Gamma$ has no 3 -tailed triangle for otherwise $\Gamma_{2}$ must have a hexagon contradicting Lemma 3.4. Suppose $\Gamma$ has no 1-tailed triangle. Hence, we have no induced claws with two pendants, which implies that $\Gamma$ has only one induced claw along with only one 2-tailed triangle as drawn in Figure 3.7.1, where $a, b, d \geq 1$ and $c \geq 0$. Clearly, $c \neq 1$ for otherwise $\operatorname{deg}_{\Gamma_{2}}(u)=4$, which is impossible. A simple verification shows that $d_{\Gamma}$ (triangle, claw $)=c$ and

$$
d_{\Gamma_{2}}(\text { triangle, claw })= \begin{cases}\frac{c+4}{2}, & c \text { is even, } \\ \frac{c-3}{2}, & c \text { is odd } .\end{cases}
$$

Since $\Gamma \cong \Gamma_{2}$ this implies that $c=4$. On the other hand, we know that

$$
|E(\Gamma)|=a+b+c+d+4
$$

and

$$
\left|E\left(\Gamma_{2}\right)\right|=a+b+c+d+5-\left[\frac{1}{d}\right]
$$

when $c \geq 2$. But then $d=1$ and $a \pm 1, b \mp 1=2,3$, from which it follows that $\Gamma_{2} \neq \Gamma$, a contradiction.

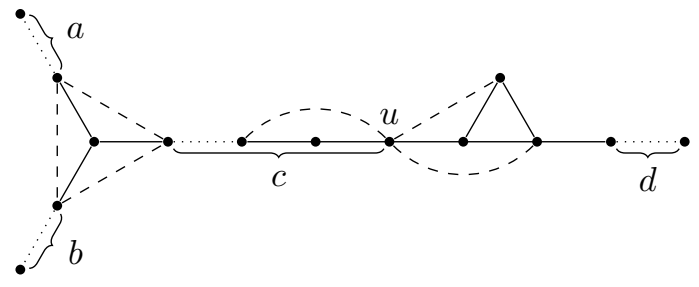


Figure 3.7.1

Therefore, $\Gamma$ has a 1-tailed triangle. Such a triangle arises from an induced claw with two pendants in $\Gamma$. Since $\Gamma$ has exactly three pendants, it can be drawn in the plane (see Figure 3.7.2) with one further triangle having an edge in the dotted areas, where $a, b \geq 0$ and $c \geq 1$ denote the number of vertices in the corresponding dotted areas. We note that every triangle in $\Gamma_{2}$ arises from an induced claw in $\Gamma$. A simple verification shows that

$$
|E(\Gamma)|=a+b+c+9
$$

and

$$
\left|E\left(\Gamma_{2}\right)\right|=a+b+c+8+\left[\frac{1}{a+1}\right]+\left[\frac{1}{b+1}\right],
$$

which implies that $a b=0$. Clearly, $c=1$ for otherwise $\operatorname{deg}_{\Gamma_{2}}(o) \geq 4$, which is impossible.

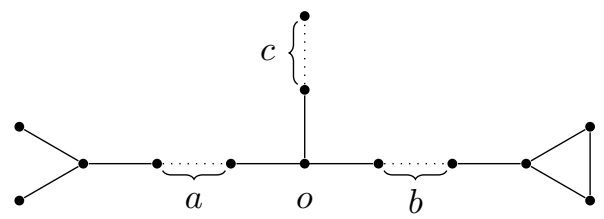

Figure 3.7.2

First assume that $a=0$. Then the graph $\Gamma$ can be drawn as in Figure 3.7.3. Note that $|A| \geq 3$ for otherwise $A$ has a vertex of degree $\geq 4$ in $\Gamma_{2}$, which is impossible. This implies that two triangles in $\Gamma_{2}$ are at distance at least five and so we must have $|B| \geq 4$. But then we obtain three induced claws in $\Gamma_{2}$ as drawn in Figure 3.7.3 with dashes, which is a contradiction.

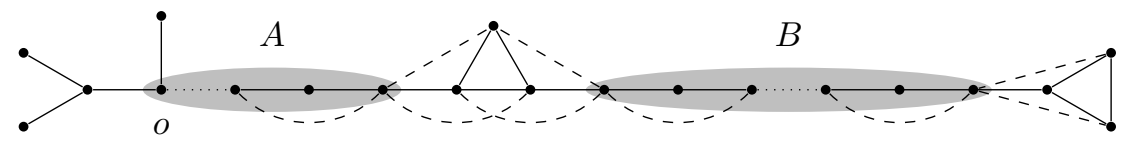

Figure 3.7.3

Finally assume that $b=0$. If $|A|=1$, then two induced claws are connected with two triangles with distance zero while it is not true in $\Gamma_{2}$. Hence, $|A| \geq 2$. If $|A|=2$, then $A$ has a vertex of degree four in $\Gamma_{2}$, which is impossible. Thus $|A| \geq 3$. Similarly, $|B| \geq 3$ for otherwise it has a vertex of degree four in $\Gamma_{2}$, a contradiction. But then we obtain three induced claws in $\Gamma_{2}$ as drawn in Figure 3.7.4 with dashes, which is a contradiction.

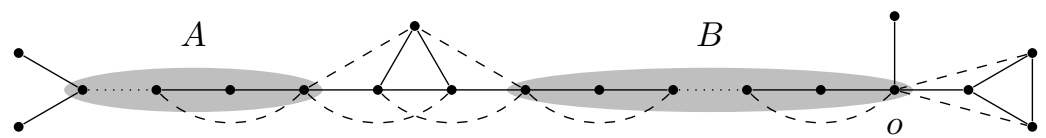

Figure 3.7.4

The proof is complete. 


\section{Graphs With DisJoint TRIANGLES}

Throughout this section, we assume that $\Gamma \cong \Gamma_{2}$ is a graph with disjoint triangles. Further we assume that $\Gamma$ is not an odd cycle. As in section 3, we proceed by analysing the existence of special subgraphs in $\Gamma$ is several lemmas. The following lemma is crucial in the proof of our results.

Lemma 4.1. We have $\Delta(\Gamma)=3$.

Proof. Let $v$ be a vertex of $\Gamma$. Clearly, $N_{\Gamma}(v)$ is a union of isolated vertices and at most one edge. Now, since $N_{\Gamma}(v)^{c}$ is a subgraph of $\Gamma_{2}$, we must have $\left|N_{\Gamma}(v)\right| \leq 3$, as required.

Lemma 4.2. If $\Gamma$ has a $C_{5} \mid C_{3}$ subgraph, then $\Gamma$ is isomorphic to $C_{5} \mid C_{3}$.

Proof. Suppose on the contrary that $\Gamma$ is not isomorphic to $C_{5} \mid C_{3}$ and consider a subgraph $S$ of $\Gamma$ isomorphic to $C_{5} \mid C_{3}$ as drawn in Figure 3.1.1. We proceed in two steps.

Case 1. The jaws are non-adjacent. Hence there is a vertex in $\Gamma \backslash S$ adjacent to some vertex of $S$. First suppose that the chin $d$ is adjacent to some new vertex $g$. If $g$ is not adjacent to jaws $c$ and $e$, then we have two triangles $\{a, c, e\}$ and $\{c, e, g\}$ with a common edge in $\Gamma_{2}$ contradicting the assumption. Hence, $g$ is adjacent to exactly one of the jaws, say $c$. But then we have two triangles $\{a, c, e\}$ and $\{b, e, g\}$ in $\Gamma_{2}$ with a common vertex, which is another contradiction. Therefore, $N_{\Gamma}(d)=\{c, e\}$. Next assume that a jaw, say $c$, is adjacent to a new vertex $g$. Clearly, $b, d, f \notin N_{\Gamma}(g)$. If $g$ and $e$ are adjacent, then we have two triangles $\{b, d, g\}$ and $\{d, f, g\}$ with a common edge in $\Gamma_{2}$, a contradiction. Hence $N_{S}(g)=\{c\}$ and the subgraph induce by $\{a, b, c, d, e, f\}$ in $\Gamma_{2}$ is isomorphic to $C_{5} \mid C_{3}$ with $g$ adjacent to its chin, which is impossible by the previous discussions. Clearly the temples are not adjacent to any vertex of $\Gamma \backslash S$. Hence, the forehead $a$ must be adjacent to a new vertex $g$ so that the subgraph induced by $\{a, b, c, d, e, f\}$ in $\Gamma_{2}$ is isomorphic to $C_{5} \mid C_{3}$ with $g$ adjacent to its jaws, which is impossible by previous arguments.

Case 2. The jaws are adjacent. Since the subgraph induced by $S$ is not a self 2-distance graph, one of the foreheads or chin must be adjacent to a new vertex $g$, say $a$ and $g$ are adjacent. Then we have two triangles $\{c, d, e\}$ and $\{c, e, g\}$ in $\left(\Gamma_{2}\right)_{2}$, which is a contradiction.

Lemma 4.3. The graph $\Gamma$ does not have any hexagon.

Proof. Suppose on the contrary that $\Gamma$ has a hexagon $S$ as in Figure 4.3 .1 with vertices $a, b, c, d, e, f$. Since there is no subgraph isomorphic to $C_{5} \mid C_{3}$ in $\Gamma$, the only possible chords of $S$ are $\{a, d\},\{b, e\}$ or $\{c, f\}$. Since $S$ is not a self 2 -distance graph, we may assume that $a$ is adjacent to a new vertex $g$. Clearly, $g$ is adjacent to exactly one of $b$ or $f$, say $b$, for otherwise either $\Gamma$ or $\Gamma_{2}$ has two triangles with a common edge. Now, by using Lemma 4.2 one can easily see that the vertices $a, b, c, d, e, f, g$ induce a subgraph in $\Gamma_{2}$ as drawn with dashes in Figure 4.3.1. Hence, the degree of $g$ in $\left(\Gamma_{2}\right)_{2}$ is at least four, which is a contradiction. 


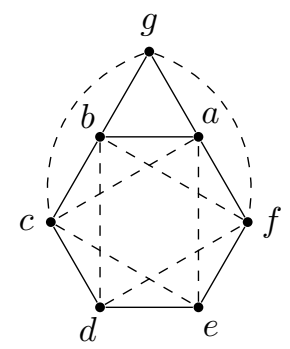

Figure 4.3.1

Lemma 4.4. The graph $\Gamma$ does not have any pentagon.

Proof. Suppose on the contrary that $\Gamma$ has a pentagon $S$ with vertices $a, b, c, d, e$. We consider two cases:

Case 1. $S$ does not have any chord. Since $\Gamma$ is not an odd cycle, we may assume that $a$ is adjacent to a new vertex $f$. By Lemma 4.2, $f$ is not adjacent to $b$ and $e$, from which it follows that $\Gamma_{2}$ has a subgraph isomorphic to $C_{5} \mid C_{3}$, a contradiction.

Case 2. $S$ has a chord. Clearly, $S$ has a unique chord, say $\{b, e\}$. Since $S$ is not a self 2-distance graph, it has a vertex adjacent to a new vertex $f$. First suppose that $a$ and $f$ are adjacent. Since $\Gamma_{2}$ does not have a subgraph isomorphic to $C_{5} \mid C_{3}$, either $c, d \in N_{\Gamma}(f)$ or $c, d \notin N_{\Gamma}(f)$. In both cases, the vertices $a, b, c, d, e, f$ induce a hexagon in $\Gamma_{2}$, contradicting Lemma 4.3 (see Figure 4.4.1). Therefore, $f$ is adjacent to $c$ or $d$, say $c$. Clearly, $N_{S}(f)=\{c\}$. If there is a vertex $g$ adjacent to $d$, then again $N_{S}(g)=\{d\}$. Now, by using Lemma 4.2, one can easily see that the vertices $a, b, c, d, e, f, g$ induce a subgraph in $\Gamma_{2}$ as drawn with dashes in Figure 4.4.2. Hence, the degree of $a$ in $\left(\Gamma_{2}\right)_{2}$ is at least four, which is a contradiction. Therefore, $d$ is not adjacent to vertices other than $c$ and $e$. This implies that the vertex $f$ is adjacent to another vertex $g$ as in Figure 4.4.3. Again, by using Lemma 4.2, the vertices $a, b, c, d, e, f, g$ induce a subgraph in $\Gamma_{2}$ as drawn with dashes in Figure 4.4.3. Hence, the degree of $a$ in $\left(\Gamma_{2}\right)_{2}$ is at least four, which is a contradiction. The proof is complete.

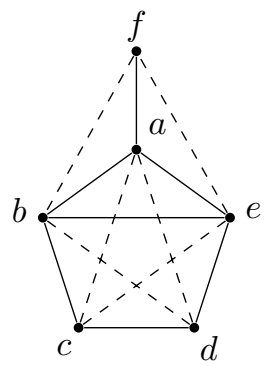

Figure 4.4.1

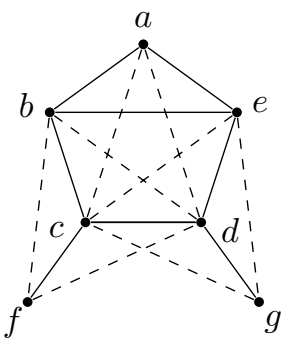

Figure 4.4.2

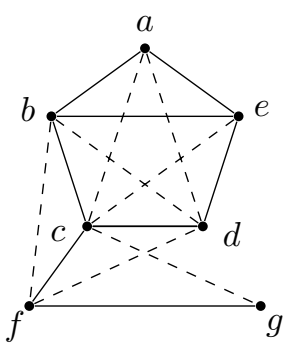

Figure 4.4.3

Lemma 4.5. The graph $\Gamma$ does not have any heptagon.

Proof. Suppose on the contrary that $\Gamma$ has a heptagon $S$ with vertices $a, b, c, d, e, f, g$. By Lemmas 4.4 and 4.3. $S$ is an induce subgraph. Since $\Gamma$ is not an odd cycle, there is a new vertex $h$ adjance to some vertex of $S$. A simple verification shows that $h$ is adjacent to two consecutive vertices of $S$ in $\Gamma$ or $\Gamma_{2}$. Hence, we may assume that $h$ is adjacent to vertices $d$ and $e$ of $S$ in $\Gamma$. By Lemmas 4.3 and 4.4 , one gets 
$N_{S}(h)=\{d, e\}$. By the same reasons, one can easily show that the subgraph of $\Gamma_{2}$ induced by the vertices $a, b, c, d, e, f, g, h$ is as drawn in Figure 4.5.1 with dashed lines. But then $\left(\Gamma_{2}\right)_{2}$ has two triangles $\{a, e, h\}$ and $\{a, d, h\}$ with a common edge, which is a contradiction.

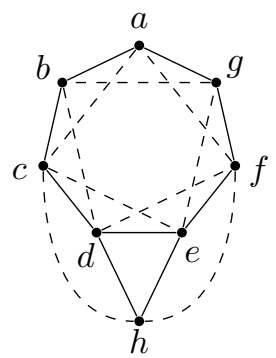

Figure 4.5.1

Lemma 4.6. The graph $\Gamma$ does not have any octagon.

Proof. Suppose on the contrary that $\Gamma$ has an octagon $S$ with $a, b, c, d, e, f, g, h$ as its vertices. By Lemmas 4.4, 4.3 and 4.5, $S$ is an induced subgraph of $\Gamma$. Since $\Gamma$ is not an even cycle, there is a new vertex $i$ adjacent to some vertex of $S$. Clearly, $i$ is adjacent to two consecutive vertices of $S$ for otherwise we have a pentagon in $\Gamma_{2}$ contradicting Lemma 4.4. Hence, we may assume that $i$ is adjacent to vertices $d$ and $e$ of $S$. Now, by Lemma 4.4, one can easily show that the subgraph of $\Gamma_{2}$ induced by the vertices $a, b, c, d, e, f, g, h, i$ is as drawn in Figure 4.6.1. But then $i$ is adjacent to vertices $a, d, e, h$ in $\left(\Gamma_{2}\right)_{2}$ contradicting Lemma 4.1. The proof is complete.

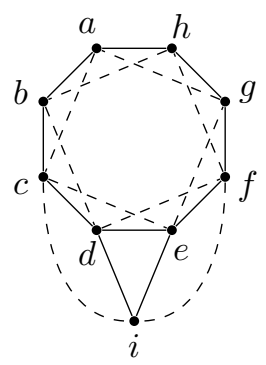

Figure 4.6.1

Theorem 4.7. Let $\Gamma$ be a self 2-distance graph with disjoint triangles. Then either $\Gamma$ is an odd cycle or it is the edged product $C_{5} \mid C_{3}$.

Proof. A simple verification shows that squares in $\Gamma_{2}$ arises from hexagons or octagons. Hence, by Lemmas 4.3 and 4.6, $\Gamma$ has no squares and the result follows by Theorem 3.7.

Corollary 4.8. There is no cubic self 2-distance graph.

Proof. By Theorem 4.7, and the fact that $\Gamma$ is not the complete graph on four vertices, it follows that $\Gamma$ has an induced subgraph as in Figure 4.8.1. Then $\operatorname{deg}_{\Gamma_{2}}(u)$ is two, which is a contradiction. 


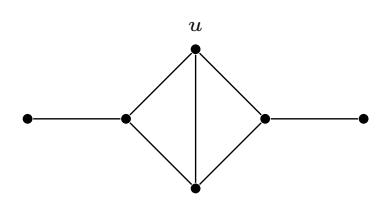

Figure 4.8.1

\section{GRAPHS WITH NO DIAMOND SUBGRAPHS}

In this section, we go further into the study of self 2-distance graphs with a forbidden subgraph, which relies on our earlier results. Remind that a diamond is the edged product of two triangles, namely $C_{3} \mid C_{3}$. A diamond with vertices $a, b$ of degree three and vertices $c, d$ of degree two is denoted by $\mathcal{D}(a, b ; c, d)$.

Theorem 5.1. Let $\Gamma$ be a self 2-distance graph with no diamond as subgraph. Then either $\Gamma$ is an odd cycle, it is the edged product $C_{5} \mid C_{3}$, or it is isomorphic to one the following graphs:

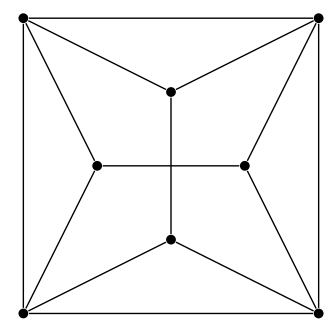

Figure 5.1.1

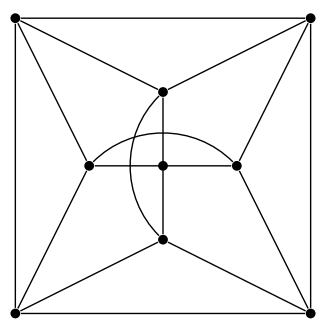

Figure 5.1.2

Proof. First we observe that $\Delta(\Gamma) \leq 4$. Indeed, if $v \in V(\Gamma)$ is an arbitrary vertex, then by assumption the subgraph induced by $N_{\Gamma}(v)$ is a union of disjoint edges and isolated vertices. On the other hand, $N_{\Gamma}(v)^{c}$ is a subgraph of $\Gamma_{2}$, from which it follows that $\left|N_{\Gamma}(v)\right| \leq 4$. If $\Delta(\Gamma) \leq 3$, then all triangles in $\Gamma$ are disjoint and the result follows by Theorem 4.7. Hence, in what follows, we assume that $\Delta(\Gamma)=4$ and that $v \in V(\Gamma)$ is a vertex of degree four. Clearly, $N_{\Gamma}(v)$ is a union of two disjoint edges, say $\{a, b\}$ and $\{c, d\}$, and that $N_{\Gamma}(a) \cap N_{\Gamma}(b)=N_{\Gamma}(c) \cap N_{\Gamma}(d)=\{v\}$. Let $X=\{a, b\}, Y=\{c, d\}$ and $M_{\Gamma}(v)$ be the set of all vertices of $\Gamma \backslash\{v\}$ adjacent to an element of $X$ and an element of $Y$. Suppose further that $\left|M_{\Gamma}(v)\right|$ is maximum among all vertices of degree four. We proceed in some steps:

Case 1. If $e, f \in M_{\Gamma}(v)$, then $N_{N_{\Gamma}(v)}(e) \neq N_{N_{\Gamma}(v)}(f)$. If not $\left(N_{\Gamma}(v) \cup\{e, f\}\right) \backslash$ $N_{N_{\Gamma}(v)}(e)$ has a diamond subgraph in $\Gamma_{2}$, which is a contradiction.

Case 2. If $e, f \in M_{\Gamma}(v)$, then $N_{\Gamma}(e), N_{\Gamma}(f) \subseteq N_{\Gamma}(v)$. If not we may assume that $e$ is adjacent to a new vertex $g$. First assume that $N_{N_{\Gamma}(v)}(e) \cap N_{N_{\Gamma}(v)}(f)=\emptyset$ and we may assume that $a, c \in N_{\Gamma}(e)$ and $b, d \in N_{\Gamma}(f)$. Then we have a diamond $\mathcal{D}(e, g ; a, c)$ in $\Gamma$ or a diamond $\mathcal{D}(a, c ; f, g)$ in $\Gamma_{2}$ according to $g$ is adjacent simultaneously to $a$ and $c$ or not, which is a contradiction. Hence $g$ is adjacent to exactly one of $a$ or $c$, say $a$. Hence $g$ is not adjacent to $d$ by assumption, from which it follows that $g$ and $f$ are not adjacent for otherwise we get a diamond $\mathcal{D}(b, d ; e, g)$ in $\Gamma_{2}$. Now, by replacing $\Gamma, v, a, b, c, d, e, f, g$ by $\Gamma_{2}, b, c, g, d, e, a, v, f$, we observe that $g$ and $f$ are adjacent, which is impossible. Thus $N_{N_{\Gamma}(v)}(e) \cap N_{N_{\Gamma}(v)}(f) \neq \emptyset$. Assume that $a, c \in N_{\Gamma}(e)$ and $a, d \in N_{\Gamma}(f)$. Then $\operatorname{deg}_{\Gamma}(a)=4$, which implies that $e$ and $f$ are adjacent. Hence $\operatorname{deg}_{\Gamma}(e)=4$ so that $c$ and $g$ are adjacent too. Now, 
by replacing $\Gamma, v, a, b, c, d, e, f$ by $\Gamma_{2}, b, e, d, f, c, v, a$, respectively, we observe that $N_{N_{\Gamma}(v)}(e) \cap N_{N_{\Gamma}(v)}(f)=\emptyset$, which is impossible as mentioned before.

Case 3. $\left|M_{\Gamma}(v)\right|=4$. We may assume that $N_{\Gamma}(a) \cap N_{\Gamma}(c)=\{v, e\}, N_{\Gamma}(b) \cap$ $N_{\Gamma}(d)=\{v, f\}, N_{\Gamma}(b) \cap N_{\Gamma}(c)=\{v, g\}$ and $N_{\Gamma}(a) \cap N_{\Gamma}(d)=\{v, h\}$ for some distinct vertices $e, f, g, h$ different from $v, a, b, c, d . \quad \operatorname{As}_{\operatorname{deg}_{\Gamma}}(a)=\operatorname{deg}_{\Gamma}(b)=\operatorname{deg}_{\Gamma}(c)=$ $\operatorname{deg}_{\Gamma}(d)=4$, the subgraph induced by $e, f, g, h$ is the 4 -cycle $\{e, g, f, h, e\}$. Hence, by using case 2, the graph $\Gamma$ is isomorphic to that drawn in Figure 5.1.2.

Case 4. $\left|M_{\Gamma}(v)\right|=3$. We may assume that $N_{\Gamma}(a) \cap N_{\Gamma}(c)=\{v, e\}, N_{\Gamma}(b) \cap$ $N_{\Gamma}(d)=\{v, f\}, N_{\Gamma}(b) \cap N_{\Gamma}(c)=\{v, g\}$ for some distinct vertices $e, f, g$ different from $v, a, b, c, d$. Since $\operatorname{deg}_{\Gamma}(b)=\operatorname{deg}_{\Gamma}(c)=4, g$ is adjacent to $e$ and $f$. But then $e$ and $f$ are not adjacent for otherwise we obtain a diamond $\mathcal{D}(e, g ; c, f)$. If $\Gamma$ has more than eight vertices, then there exists a new vertex $h$ adjacent to $a$ or $d$, say $a$. Since $\operatorname{deg}_{\Gamma}(a)=4, e$ and $h$ must be adjacent. Then $h$ is adjacent to $b, c, v$ in $\Gamma_{2}$ so that $\left|M_{\Gamma_{2}}(v)\right|=4$. Hence, by case $3, \Gamma$ is isomorphic to the graph in Figure 5.1.2, which is a contradiction. Therefore, the only vertices of $\Gamma$ are $v, a, b, c, d, e, f, g$ and $\Gamma$ is isomorphic to the graph drawn in Figure 5.1.1.

Case 5. $\left|M_{\Gamma}(v)\right|=2$. Then $M_{\Gamma}(v)=\{e, f\}$ for some vertices $e$ and $f$. First assume that $N_{N_{\Gamma}(v)}(e) \cap N_{N_{\Gamma}(v)}(f)=\emptyset$, say $N_{N_{\Gamma}(v)}(e)=\{a, c\}$ and $N_{N_{\Gamma}(v)}(f)=$ $\{b, d\}$. By case 2 , there exists a new vertex $g$ adjacent to $a, b, c$ or $d$, say $a$. Then $\operatorname{deg}_{\Gamma}(a)=4$, which implies that $g$ and $e$ are adjacent, contradicting case 2 . Thus $N_{N_{\Gamma}(v)}(e) \cap N_{N_{\Gamma}(v)}(f) \neq \emptyset$, say $N_{N_{\Gamma}(v)}(e)=\{a, c\}$ and $N_{N_{\Gamma}(v)}(f)=\{a, d\}$. Then $M_{\Gamma_{2}}(b)=\{a, v\}$ and $N_{N_{\Gamma}(b)}(a) \cap N_{N_{\Gamma}(b)}(v)=\emptyset$, which is impossible by similar arguments as before.

Case 6. $\left|M_{\Gamma}(v)\right|=1$. Suppose that $M_{\Gamma}(v)=\{e\}$ and $N_{N_{\Gamma}(v)}(e)=\{a, c\}$. First, we observe that neither $a$ nor $c$ is adjacent to a new vertex. If not we may assume that $a$ is adjacent to a new vertex $f$, from which it follows $e$ and $f$ must be adjacent. But then $a, v \in M_{\Gamma_{2}}(b)$ contradicting the choice of $v$ as $\Gamma_{2} \cong \Gamma$. Now, if two new vertices $f$ and $g$ are adjacent to $b$ or $d$, say $b$, then $\operatorname{deg}_{\Gamma_{2}}(a)=4$ while $b$ is an isolated vertex in $N_{\Gamma_{2}}(a)=\{c, d, f, g\}$, which is a contradiction. Hence, we may assume that neither $b$ nor $d$ is adjacent to two vertices other than $v, a, c$. Next assume that $b$ and $d$ are adjacent to new vertices $f$ and $g$, respectively. If $f$ and $g$ are adjacent, then $a, v \in M_{\Gamma_{2}}(b)$, contradicting the choice of $v$. Hence, assume that $f$ and $g$ are not adjacent and consequently $b$ and $d$ are not adjacent to $g$ and $f$ in $\Gamma_{2}$, respectively. Also, $a$ and $g$ are not adjacent in $\Gamma_{2}$ for otherwise $d$ and $f$ must be adjacent in $\Gamma_{2}$, which is impossible. Now, it is easy to see that $\left(\Gamma_{2}\right)_{2}$ has a diamond $\mathcal{D}(a, b ; g, v)$, which is a contradiction. Hence, we may assume that at most one of $b$ and $d$ are adjacent to a new vertex. Suppose $b$ is such an element adjacent to a new vertex $f$. Then either we have a diamond $\mathcal{D}(c, d ; f, v)$ in $\left(\Gamma_{2}\right)_{2}$ when $c$ and $f$ are not adjacent in $\Gamma_{2}$, or $e, f \in M_{\left(\Gamma_{2}\right)_{2}}(v)$ when $c$ and $f$ are adjacent in $\Gamma_{2}$, which is a contradiction. Therefore, neither $b$ nor $d$ is adjacent to a new vertex other than $v, a, c$. Then, the second neighborhood of $v$ is consists of $e$ only. Since the subgraph induced by $a, b, c, d, e$ is not self 2-distance graph, the vertex $e$ must be adjacent to some vertices other than $v, a, b, c, d$. If $e$ is adjacent to two new vertices $f, g$, then $\Gamma_{2}$ has a diamond $\mathcal{D}(a, c ; f, g)$, which is a contradiction. Hence, $N_{\Gamma}(e)=\{a, c, f\}$ for some vertex $f$. As $b, d, v \in N_{\Gamma_{2}}(e)$ and $\operatorname{deg}_{\Gamma_{2}}(e) \leq 4$, there must exists another vertex $g$ such that $N_{\Gamma}(f)=\{e, g\}$. Then $N_{\Gamma_{2}}(e)=\{b, d, v, g\}$ so that $v$ and $g$ must be adjacent in $\Gamma_{2}$, which is impossible as $d_{\Gamma}(v, g)=4$. 
Case 7. $M_{\Gamma}(v)=\emptyset$. First suppose that three vertices among $a, b, c, d$ are adjacent to new vertices, say $a, b, c$ are adjacent to distinct vertices $e, f, g$, respectively. If $g$ is adjacent to $e$ or $f$, say $e$, then $N_{\Gamma_{2}}(a)=\{c, d, f, g\}$ and hence $c$ and $f$ must be adjacent in $\Gamma_{2}$, that is, $c$ and $f$ are connected in $\Gamma$ via a path of length 2 . Clearly, $f$ and $g$ are not adjacent for otherwise we have a diamond $\mathcal{D}(d, g ; a, b)$ in $\Gamma_{2}$, a contradiction. Hence, there exists a new vertex $h$ adjacent to both $c$ and $f$. Then $N_{\Gamma}(c)=\{v, d, g, h\}$ so that $g$ and $h$ must be adjacent. But then $f$ and $g$ are adjacent in $\Gamma_{2}$, which results in a diamond $\mathcal{D}(a, f ; c, g)$ in $\Gamma_{2}$, a contradiction. Thus, we deduce that there is no edges from $N_{\Gamma}(a) \cup N_{\Gamma}(b)$ to $N_{\Gamma}(c) \cup N_{\Gamma}(d)$, from which we obtain a diamond $\mathcal{D}(a, b ; v, g)$ in $\left(\Gamma_{2}\right)_{2}$, a contradiction. Next assume that exactly two vertices among $a, b, c, d$ are adjacent to vertices other than $v, a, b, c, d$. We have two cases up to symmetry:

(i) $a$ and $b$ are adjacent to two distinct new vertices $e$ and $f$, respectively. If $e$ or $f$, say $e$, is adjacent to another vertex $g$ in the third neighborhood of $v$, then $N_{\Gamma_{2}}(a)=$ $\{c, d, f, g\}$ where $\{c, d, f\}$ induces an independent set in $\Gamma_{2}$, a contradiction. On the other hand, if $a$ or $b$, say $a$, is adjacent to another vertex $g$, then $N_{\Gamma_{2}}(b)=\{c, d, e, g\}$ with $\{c, d, e\}$ an independent set in $\Gamma_{2}$, which is again a contradiction.

(ii) $a$ and $c$ are adjacent to two distinct new vertices $e$ and $f$, respectively. If $e$ and $f$ are adjacent, then $e, f \in M_{\left(\Gamma_{2}\right)_{2}}(v)$, which contradicts the choice of $v$ as $\left(\Gamma_{2}\right)_{2} \cong \Gamma$. Hence, we may assume that there is no edges from $N_{\Gamma}(a) \backslash\{v, b\}$ to $N_{\Gamma}(c) \backslash\{v, d\}$. If $e$ is adjacent to a new vertex $g$, then $N_{\left(\Gamma_{2}\right)_{2}}(d)=\{c, e, g, v\}$ with $\{c, g, v\}$ an independent set in $\left(\Gamma_{2}\right)_{2}$, which is impossible. Hence, $N_{\Gamma}(e)=\{a\}$ and similarly $N_{\Gamma}(f)=\{c\}$. Since, the subgraph induced by $v, a, b, c, d, e, f$ is not a self 2-distance graph, we may assume that $a$ is adjacent to another vertex $g$. But then $e$ and $g$ are adjacent and hence $N_{\Gamma_{2}}(b)=\{c, d, e, g\}$ with $\{d, e, g\}$ an independent subset in $\Gamma_{2}$, which is a contradiction.

Finally, assume that only one of the vertices $a, b, c, d$ is adjacent to a vertex other than $v, a, b, c, d$, say $a$ is adjacent to a new vertex $e$. If $a$ is adjacent to another vertex $f$, then as before $N_{\Gamma_{2}}(b)=\{c, d, e, f\}$ with $\{d, e, f\}$ an independent subset in $\Gamma_{2}$, which is a contradiction. Hence $N_{\Gamma}(a)=\{v, b, e\}$ so that $e$ is adjacent to a vertex $f$, from which we obtain a diamond $\mathcal{D}(c, d ; e, f)$ in $\left(\Gamma_{2}\right)_{2}$, which is a contradiction. The proof is complete.

\section{OPEN PROBLEMS}

We devote the last section of this paper to some open problems arising in our study of self 2-distance graphs. The following conjecture, if it is true, can be applied to shorten our proofs, and also will be useful while studying self 2-distance graphs with other forbidden subgraphs.

Conjecture 1. Every self 2-distance graph is 2-connected.

A graph $\Gamma$ with $v$ vertices is strongly regular of degree $k$ if there are integers $\lambda$ and $\mu$ such that every two adjacent vertices have $\lambda$ common neighbors and every two non-adjacent vertices have $\mu$ common neighbors. The numbers $v, k, \lambda, \mu$ are the parameters of the corresponding graph.

Theorem 6.1. Every strongly regular self 2-distance graphs is a self-complimentary graph and has parameters $(4 t+1,2 t, t-1, t)$ where the number of vertices is a sum of two squares. 
Proof. The result follows from [9] and the fact that every strongly regular graph has diameter at most two.

We have shown, in Corollary 4.8, that there is no self 2-distance cubic graph. Indeed, we believe that a more general case also holds for regular graphs with odd degrees while the same result cannot hold for regular graphs of even degrees by the above theorem.

Conjecture 2. There are no regular self 2-distance graphs of odd degree.

We note that if the above conjecture is true then for any finite group $G$ and any inversed closed subset $S$ of $G \backslash\{1\}$ of odd size, the sets $S^{2} \backslash S$ and $S$ belong to different orbits of the poset of subsets of $G$ under the action of automorphism group of $G$.

\section{REFERENCES}

[1] A. Azimi and M. Farrokhi D. G., Simple graphs whose 2-distance graphs are paths or cycles, Matematiche (Catania) 69(2) (2014), 183-191.

[2] K. B. Chilakamarri, The unit-distance graph problem: a brief survey and some new results, Bull. Inst. Combin. Appl. 8 (1993), 39-60.

[3] R. B. Eggleton, P. Erdös and D. K. Skilton, Colouring the real line, J. Combin. Theory, Ser. $B, 39(1)$ (1985), 86-100.

[4] H. Hadwiger, H. Debrunner and V. Klee, Combinatorial Geometry in the Plane, New York, Holt, Rinehart and Winston, 1964.

[5] J. Isbell and E. Nelson, Unpublished.

[6] F. Kramer and H. Kramer, A survey on the distance-colouring of graphs, Discrete Math. 308(2-3) (2008), 422-426.

[7] D. D. Liu, From rainbow to the lonely runner: a survey on coloring parameters of distance graphs, Taiwanese J. Math. 12(4) (2008), 851-871.

[8] L. Moser and W. Moser, Solution to problem 10, Canad. Math. Bull. 4 (1961), 187-189.

[9] J. J. Seidel, A survey of two-graphs in Proc. Int. Coil. Teorie Combinatorie, I (1973), Acad. Naz. Lincei (1976), 481-511.

[10] S. K. Simić, Graph Equations for line graphs and n-distance graphs, Publ. Inst. Math. (Beograd) 33(47) (1983), 203-216.

[11] A. Soifer, The Mathematical Coloring Book: Mathematics of Coloring and the Colorful Life of Its Creators, Springer, New York, 2009.

Department of Pure Mathematics, Ferdowsi University of Mashhad, Mashhad, Iran.

E-mail address: ali.azimi61@gmail.com

Mathematical Science Research Unit, College of Liberal Arts, Muroran Institute of Technology, 27-1, Mizumoto, Muroran 050-8585, Hokkaido, Japan.

E-mail address: m.farrokhi.d.g@gmail.com 